\title{
Melatonin prevents inflammation and oxidative stress caused by abdominopelvic and total body irradiation of rat small intestine
}

Y. Guney ${ }^{1}$, A. Hicsonmez ${ }^{1}$, C. Uluoglu², H.Z. Guney², U. Ozel Turkcu ${ }^{5}$, G. Take ${ }^{3}$, B. Yucel ${ }^{2}$, G. Caglar ${ }^{3}$, A. Bilgihan ${ }^{4}$, D. Erdogan ${ }^{3}$, M. Nalca Andrieu ${ }^{1}$, C. Kurtman ${ }^{1}$ and H. Zengil ${ }^{2}$

\author{
'Department of Radiation Oncology, Ankara University Faculty of Medicine, \\ Ankara, Turkey \\ ${ }^{2}$ Department of Pharmacology, ${ }^{3}$ Department of Histology, \\ ${ }^{4}$ Department of Biochemistry, Gazi University Faculty of Medicine, Ankara, Turkey \\ ${ }^{5}$ Mugla School of Health Sciences, Mugla University, Mugla, Turkey
}

\section{Correspondence \\ Y. Guney \\ Department of Radiation Oncology, Ankara University School of Medicine Cebeci Hospital \\ Dikimevi, Ankara \\ 06100 Turkey \\ Fax: +90-312-362-1495 \\ E-mail: yildiz_guney@yahoo.com}

Received October 21, 2006

Accepted May 14, 2007

\begin{abstract}
We investigated the day-night differences in intestinal oxidativeinjury and the inflammatory response following total body (TB) or abdominopelvic (AP) irradiation, and the influence of melatonin administration on tissue injury induced by radiation. Rats (male Wistar, weighing 220-280 g) in the irradiated groups were exposed to a dose of $8 \mathrm{~Gy}$ to the TB or AP region in the morning (resting period $-1 \mathrm{~h}$ after light onset) or evening (activity span - $13 \mathrm{~h}$ after light onset). Vehicle or melatonin was administered immediately before, immediately after and $24 \mathrm{~h}$ after irradiation $(10,2.0$ and $10 \mathrm{mg} / \mathrm{kg}$, $i p$, respectively) to the irradiated rats. AP $(\mathrm{P}<0.05)$ and $\mathrm{TB}(\mathrm{P}<0.05)$ irradiation applied in the morning caused a significant increase in thiobarbituric acid reactive substance (TBARS) levels. Melatonin treatment in the morning $(\mathrm{P}<0.05)$ or evening $(\mathrm{P}<0.05)$ decreased TBARS levels after TB irradiation. After AP irradiation, melatonin treatment only in the morning caused a significant decrease in TBARS levels $(\mathrm{P}<0.05)$. Although we have confirmed the development of inflammation after radiotherapy by histological findings, neither AP nor TB irradiation caused any marked changes in myeloperoxidase activity in the morning or evening. Our results indicate that oxidative damage is more prominent in rats receiving $\mathrm{TB}$ and $\mathrm{AP}$ irradiation in the morning and melatonin appears to have beneficial effects on oxidative damage irrespective of the time of administration. Increased neutrophil accumulation indicates that melatonin administration exerts a protective effect on AP irradiation-induced tissue oxidative injury, especially in the morning.
\end{abstract}

Key words

- Irradiation

- Melatonin

- Circadian rhythm

- Oxidative stress

- Thiobarbituric acid reactive substances

- Myeloperoxidase 


\section{Introduction}

Radiation therapy (RT) is considered to be one of the most popular and important therapeutic modalities for the cure of cancer (1) and is the mainstay in the treatment of abdominopelvic cancers (2). The intestine is highly radiosensitive and is an important dose-limiting organ when RT is applied either to the total body (TB) or only to the abdominopelvic (AP) region, since intestinal epithelial cells have high proliferation rates (3). Application of RT in abdominal and pelvic malignancies causes radiation enteropathy (4) and this adverse effect is usually attributed to the loss of epithelial integrity following TB (5) or abdominal irradiation (6). This breakdown in the mucosa is preceded by an acute abdominal inflammation (7).

Radiation-induced lipid peroxidation is a free radical process (8) that involves oxidative conversion of polyunsaturated fatty acids to several products including malondialdehyde (MDA) and lipid peroxides (9).

Radiation induces an inflammatory response in the target and surrounding normal tissues, and accumulation of leukocytes (10). Thiobarbituric acid reactive substances (TBARS) and myeloperoxidase (MPO) play a fundamental role in oxidant production by neutrophils, and cause tissue damage (11). It has been shown that MPO activity significantly increases in the ileum of ferrets 2 days after AP irradiation, but decreases after TB irradiation (12).

It has been reported that melatonin (Nacetyl-5-methoxytryptamine) is mainly produced in the pineal gland in addition to the gastrointestinal system, and has been considered to be a component of the antioxidant defense system. This hormone directly neutralizes a number of toxic reactants and stabilizes cell membranes, making them more resistant to oxidative attack $(13,14)$.

Several body functions follow distinct day/night rhythms and are controlled by the circadian system (15). Plasma levels of melatonin exhibit a circadian variation, with the highest concentrations occurring at night and lower levels during the day (16). Light exposure suppresses melatonin secretion (17). Rhythms in free radical formation by leukocytes have been demonstrated in mice (18, 19), particularly during inflammation. The highest toxicity after TB irradiation in 12:12$\mathrm{h}$ synchronized animals was shown during the daily activity span in mice and rats (20). Circadian variations in the activities of 6phosphogluconate dehydrogenase in the liver of control and streptozotocin-induced diabetic rats (21) and temporal variations in hepatic superoxide dismutase activity have been demonstrated in mice (22). Additionally, circadian variations in the activity of superoxide dismutase in the pineal gland of rats have been demonstrated (23).

The aim of the present study was to investigate the day-night differences in the intestinal oxidative injury, as well as the inflammatory response following TB or AP irradiation, the influence of melatonin administration on tissue injury, and the oxidative damage induced by RT.

\section{Material and Methods}

\section{Experimental design}

The experiments were performed on male Wistar rats weighing 220-280 g, which were kept at a constant temperature of $22 \pm 1^{\circ} \mathrm{C}$. The animals were fed a standard rat chow diet, had access to water ad libitum, and were synchronized by the maintenance of controlled environmental conditions (light, temperature, feeding time, etc.) for at least 2 weeks prior to, and throughout the experiments. The lighting regimen was $12 \mathrm{~h}$ of light alternating with $12 \mathrm{~h}$ of darkness (lights on from 8:00 am to 8:00 pm) with a light intensity of approximately 100 lux. Lighting was provided by cool fluorescent bulbs controlled by an automatic timer. This standard- 
ized light-dark cycle acts as an entraining agent of the circadian rhythm. After 2 weeks of such synchronization, the biology of each animal approximates the biology of the entire experimental group. The experiments were performed in the months of February and March in order to avoid the influence of seasonal rhythms on the findings.

All animal procedures were carried out in accordance with the regulations of the Local Ethics Committee. A total of 82 adult animals were synchronized in 10 groups. The rats in the irradiated groups were exposed to a sublethal irradiation dose of $8 \mathrm{~Gy}$, either to the TB or AP region after ketamine anesthesia using a ${ }^{60} \mathrm{Co}$ source at a focus of $80 \mathrm{~cm}$ away from the skin at two different times during the $24 \mathrm{~h}$. Morning irradiation was performed $1 \mathrm{~h}$ after lights on (1 HALO; at 9:00 am) and evening irradiation was performed at $13 \mathrm{~h}$ after lights on (13 HALO; at 9:00 pm) since plasma melatonin levels are expected to be higher at night. In order to provide AP irradiation to the rats, a simulation film was shot and a guarding field was formed. Melatonin was dissolved in ethanol (20\%). Melatonin or vehicle (20\% ethanol) was administered to the rats immediately before, immediately after, and $24 \mathrm{~h}$ after irradiation (melatonin dose: 10, 20, and 10 $\mathrm{mg} / \mathrm{kg}$, ip, respectively). Forty-eight hours after irradiation, all animals were sacrificed with ketamine. Intestinal tissues were removed immediately and stored at $-80^{\circ} \mathrm{C}$ until the time of measurement of TBARS levels and MPO activity. Control rats did not receive any treatment. Changes in TBARS levels, a marker of lipid peroxidation and MPO activity and an index of neutrophil infiltration, were determined in the intestinal mucosa.

\section{Experimental groups}

The experimental groups were as follows: group 1: control; group 2: total body irradiation and vehicle (ethanol) administra- tion; group 3: AP irradiation and vehicle (ethanol) administration; group 4: melatonin administration together with TB irradiation; group 5: melatonin administration together with AP irradiation. Administration was in the morning (A, 1 HALO) or in the evening (B, 13 HALO). Each group consists of 6-12 animals.

\section{Determination of thiobarbituric acid reactive levels}

TBARS levels were determined in intestinal mucosa homogenized at the ratio of $1 /$ $10(\mathrm{w} / \mathrm{v})$ in $1.15 \%$ cold $\mathrm{KCl}$ solution by the thiobarbituric acid method, and the results are reported as nmol/g tissue (24).

\section{Determination of myeloperoxidase activity}

Tissue-associated MPO activity in intestinal mucosa was determined by the method of Grisham et al. (25). Samples of intestinal mucosa weighing approximately $100 \mathrm{mg}$ were homogenized in $1 \mathrm{~mL}$ ice-cold $0.02 \mathrm{M}$ EDTA, pH 4.7, for $60 \mathrm{~s}$. One milliliter mucosa homogenate was centrifuged at 20,000 $g$ for $15 \mathrm{~min}$ at $+4^{\circ} \mathrm{C}$ to obtain the pellet of the insoluble cellular debris. The supernatant, which contained less than 5\% total MPO activity, was discarded. The pellet was then re-homogenized in an equivalent volume of $0.05 \mathrm{M}$ potassium phosphate buffer, $\mathrm{pH} 6.0$, containing $0.5 \%$ hexadecyltrimethylammonium bromide. This homogenate was centrifuged at $20,000 \mathrm{~g}$ for $15 \mathrm{~min}$ at $+4^{\circ} \mathrm{C}$ and the supernatants were used in the MPO assay. MPO activity was assessed by measuring the $\mathrm{H}_{2} \mathrm{O}_{2}$-dependent oxidation of odianisidin. One unit of enzyme activity was defined as the amount of MPO present that caused a change in absorbance of 1.0/min at $410 \mathrm{~nm}$ and $37^{\circ} \mathrm{C}(26)$.

\section{Histological analysis}

Tissue samples were fixed in $10 \%$ neu- 
tral formalin for $72 \mathrm{~h}$ and paraffin blocks were obtained and routinely processed for light microscopy. Slices of 4-5 $\mu \mathrm{m}$ were obtained from the prepared blocks and stained with hematoxylin-eosin. The preparations obtained were visualized using a Leica Digital Microscope DM 4000 B-M 4-(Leica DM4000B, Wetzlar, Germany).

\section{Statistical analysis}

Differences between groups were analyzed statistically by two-way analysis of variance (ANOVA). Post hoc tests were performed using a multiple comparison procedure (Tukey test). Statistical significance was accepted as $\mathrm{P}<0.05$.

Figure 1. Thiobarbituric acid reactive substances (TBARS) in the rat ileum after total body (TB) or abdominopelvic (AP) irradiation and vehicle or melatonin (MLT) treatment. MLT or vehicle ( $20 \%$ ethanol) was administered to the rats immediately before, immediately after, and $24 \mathrm{~h}$ after irradiation (MLT dose: 10, 20, and $10 \mathrm{mg} / \mathrm{kg}$, ip, respectively).

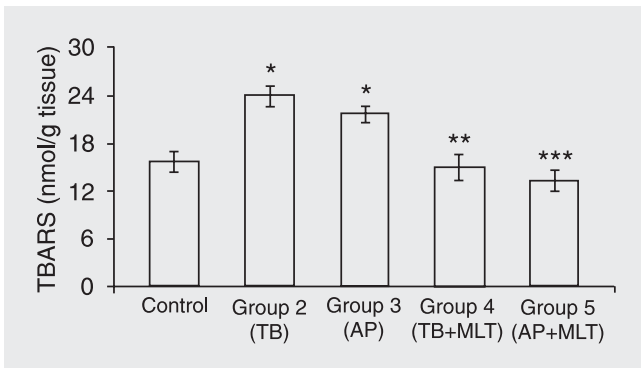
All bars represent the pooled data of rats irradiated in the morning $(A)$ and evening (B). Group 1: control; group 2: TB irradiation; group 3: AP irradiation; group 4: TB irradiation and MLT treatment; group 5: AP irradiation and MLT treatment. ${ }^{*} \mathrm{P}<0.05$ compared to control; ${ }^{* *} \mathrm{P}<0.05$ compared to group $2 ;{ }^{* * *} \mathrm{P}<0.05$ compared to group 3 . Differences between groups were analyzed statistically by two-way ANOVA. Post hoc tests were performed using a multiple comparison procedure (Tukey test).

Figure 2. Levels of thiobarbituric acid reactive substances (TBARS) in the rat ileum after total body (TB) or abdominopelvic (AP) irradiation and vehicle or melatonin (MLT) treatment in the morning $(A)$ or in the evening (B). MLT or vehicle $(20 \%$ ethanol) was administered to the rats immediately before, im-

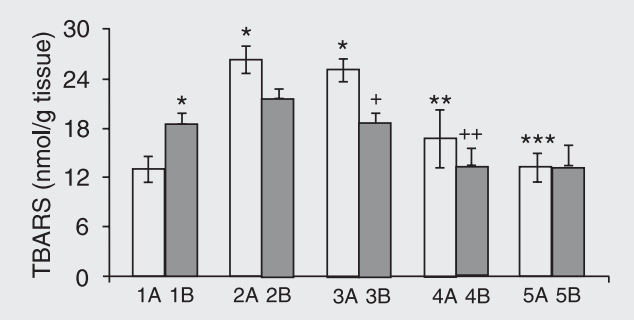
mediately after, and $24 \mathrm{~h}$ before irradiation (MLT dose: 10,20 , and $10 \mathrm{mg} / \mathrm{kg}$, ip, respectively). Group 1: control; group 2: TB irradiation; group 3: AP irradiation; group 4: TB irradiation $+\mathrm{MLT}$; group 5: AP irradiation + MLT. ${ }^{*} \mathrm{P}<0.05$ compared to group $1 \mathrm{~A},{ }^{* *} \mathrm{P}<0.05$ compared to group $2 \mathrm{~A},{ }^{* * *} \mathrm{P}<0.05$ compared to group $3 \mathrm{~A},{ }^{+} \mathrm{P}<0.05$ compared to group $3 \mathrm{~A}$, ${ }^{++} \mathrm{P}<0.05$ compared to group 2B. Differences between groups were analyzed statistically by two-way ANOVA. Post hoc tests were performed using a multiple comparison procedure (Tukey test).

\section{Results}

Thiobarbituric acid reactive substance levels

When the time of irradiation was not taken into consideration, there was a statistically significant difference $(\mathrm{P}<0.001)$ among the treatment groups (groups 1-5) determined by two-way ANOVA. TBARS levels in ileum were found to be significantly higher in rats exposed to TB (group 2) and AP (group 3) irradiation, compared to control (group 1; $\mathrm{P}$ $<0.05$ ). Melatonin treatment significantly abolished the irradiation-induced elevations in TBARS levels $(\mathrm{P}<0.05)$ in both TB(group 4) and AP- (group 5) irradiated rats (Figure 1).

Influence of time of exposure to total body irradiation on thiobarbituric acid reactive substance levels

TBARS levels in the control groups were higher in the evening than in the morning (group 1A vs 1B; $\mathrm{P}<0.05$; Figure 2). TBARS levels were found to be increased after TB irradiation (group 2A) in the morning when compared with $1 \mathrm{~A}$ and treatment with melatonin significantly decreased TBARS levels (group 2A $v s$ group $4 \mathrm{~A} ; \mathrm{P}<0.05$ ).

However, TB irradiation (group 2B) in the evening did not cause any significant changes in TBARS levels but treatment with melatonin significantly reduced TBARS levels (group 2B vs 4B; $\mathrm{P}<0.05$; Figure 2).

\section{Influence of time of exposure to} abdominopelvic irradiation on thiobarbituric acid reactive substance levels

When the influence of the time of irradiation was assessed, TBARS levels were found to be increased after AP irradiation (group $3 \mathrm{~A}$ ) in the morning compared to control (group 1A) while melatonin treatment significantly decreased ileal neutrophil infiltration induced by AP irradiation (group 3A vs 
group $5 \mathrm{~A} ; \mathrm{P}<0.05)$.

However, AP irradiation (group 3B) and melatonin treatment (group 5B) in the evening did not cause any significant changes in TBARS levels (Figure 2). TBARS levels tended to decrease but did not reach statistical significance after melatonin treatment in the evening, but were comparable to control values in AP-irradiated rats.

\section{Myeloperoxidase activity}

When the time of irradiation was not taken into account, there was a statistically significant difference $(\mathrm{P}<0.001)$ among the treatment groups (groups 1-5) determined by two-way ANOVA (Figure 3). After AP irradiation (group 3), MPO activity was markedly increased compared to control (group $1 ; \mathrm{P}<0.05$ ), while melatonin administration decreased MPO activity in rats exposed to AP irradiation (group 5; $\mathrm{P}<0.05$; Figure 3).

However, MPO activity in the ileum did not markedly change after TB irradiation (group 2) and melatonin administration did not influence MPO activity in group 4 (Figure 3 ).

Influence of the time of exposure to abdominopelvic irradiation on myeloperoxidase levels

MPO activity did not differ between the control groups (group 1A vs 1B; Figure 4). When the influence of the time of irradiation was examined, MPO activity did not change after AP irradiation (group 3A) in the morning when compared with $1 \mathrm{~A}$ but melatonin treatment caused a significant decrease in rats exposed to AP irradiation (group 3A vs group 5A; $\mathrm{P}<0.05$ ). Similarly, MPO activity did not change after AP irradiation (group $3 \mathrm{~B}$ ) in the evening when compared with $1 \mathrm{~B}$. However, MPO activity in the ileum was markedly decreased by melatonin treatment (group 3B vs group 5B; $\mathrm{P}<0.05$; Figure 4).

\section{Influence of time of exposure to total body irradiation on myeloperoxidase levels}

MPO activity did not change after TB irradiation (group 2A) in the morning compared with $1 \mathrm{~A}$. Melatonin treatment in the morning caused a significant decrease in rats exposed to TB irradiation (group 2A vs group $4 \mathrm{~A} ; \mathrm{P}<0.05)$. MPO activity was not significantly changed by TB irradiation (group 2B) in the evening compared with 1B. However, melatonin treatment in the evening caused a significant increase in rats exposed to $\mathrm{TB}$ irradiation (group 2B vs group 4B; $\mathrm{P}<0.05$; Figure 4).

\section{Histological changes following radiotherapy}

In the present study, we observed an increase in lymphatic infiltration in both TB- (group 2) or AP- (group 3) irradiated

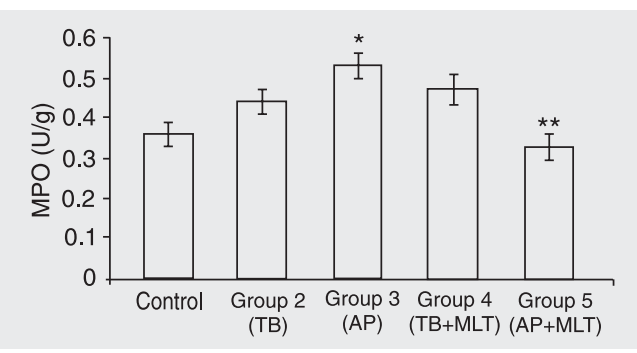

Figure 3. Myeloperoxidase (MPO) activity in the rat ileum after total body (TB) or abdominopelvic (AP) irradiation and vehicle or melatonin (MLT) treatment. All columns indicate the pooled data of rats irradiated in the morning $(A)$ and evening $(B)$. MLT or vehicle $(20 \%$ ethanol) was administered to the rats immediately before, immediately after, and $24 \mathrm{~h}$ after irradiation (MLT dose: 10, 20, and $10 \mathrm{mg} / \mathrm{kg}$, ip, respectively). Group 1: control; group 2: TB irradiation; group 3: AP irradiation; group 4: TB irradiation + MLT; group 5: AP irradiation $+\mathrm{MLT}$. ${ }^{*} \mathrm{P}<0.05$ compared to control; ${ }^{* *} \mathrm{P}<0.05$ compared to group 3 . Differences between groups were analyzed statistically by two-way ANOVA. Post hoc tests were performed using a multiple comparison procedure (Tukey test).

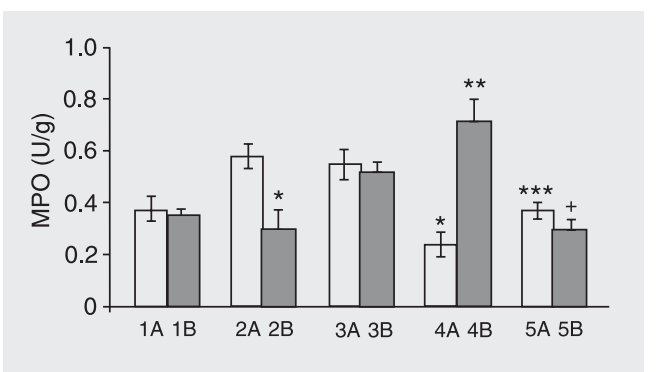

Figure 4. Myeloperoxidase (MPO) activity in the rat ileum after total body (TB) irradiation or abdominopelvic (AP) irradiation and vehicle or melatonin (MLT) treatment in the morning (A) or in the evening (B). Group 1: control; group 2: TB irradiation; group 3: AP irradiation; group 4: TB irradiation + MLT; group 5: AP irradiation + MLT. ${ }^{*} \mathrm{P}<0.05$ compared to group $2 \mathrm{~A} ;{ }^{* \star} \mathrm{P}<0.05$ compared to group $4 \mathrm{~A}$; ${ }^{\star \star *} \mathrm{P}<0.05$ compared to group $3 \mathrm{~A} ;{ }^{+} \mathrm{P}<0.05$ compared to group $3 \mathrm{~B}$. Differences between groups were analyzed statistically by two-way ANOVA. Post hoc tests were performed using a multiple comparison procedure (Tukey test). 
rats compared to control. The number of ileal villi and villus height decreased in groups $2 \mathrm{~A}$ and $2 \mathrm{~B}$, and in group $2 \mathrm{~B}$, an increase in mucin-producing goblet cells was observed (Figure 5a) when compared with group 2A (data not shown).

In rats receiving $\mathrm{AP}$ irradiation in the morning (group $3 \mathrm{~A}$ ), capillary dilatation was

Figure 5. Histological findings following radiotherapy. a, Total body irradiation group. Preparation obtained $13 \mathrm{~h}$ after light onset. $\mathrm{V}=$ irregular types of villi; asterisk $=$ goblet cells producing mucin; Gl = intestinal gland; arrow = cubic surface epithelial cells; lozenge = lymphatic region; plus symbol = dilated capillaries. $b$, Abdominopelvic irradiation group. Preparation obtained $13 \mathrm{~h}$ after light onset. $\mathrm{V}=$ completely rounded villus region; asterisk = goblet cell; $\mathrm{Gl}=$ intestinal gland; arrow = prisma surface epithelial cells; lozenge = diffuse lymphatic infiltration; plus symbol = dilatated capillaries. $c$, Total body irradiation + melatonin group. Preparation obtained $13 \mathrm{~h}$ after light onset. V = localized produced villus; asterisk $=$ few numbers of goblet cells; Gl = intestinal gland; arrow $=$ localized prevented surface epithelial cells; lozenge = diffuse lymphatic infiltration region. $d$, Abdominopelvic irradiation + melatonin group. Preparation obtained $13 \mathrm{~h}$ after light onset. V = villus; asterisk = goblet cells; $\mathrm{Gl}=$ intestinal gland; arrow = prismatic surface epithelial cell; lozenge = localized lymphatic infiltration region. $\mathrm{H} \& \mathrm{E} ; \mathrm{100X}$ for all panels.
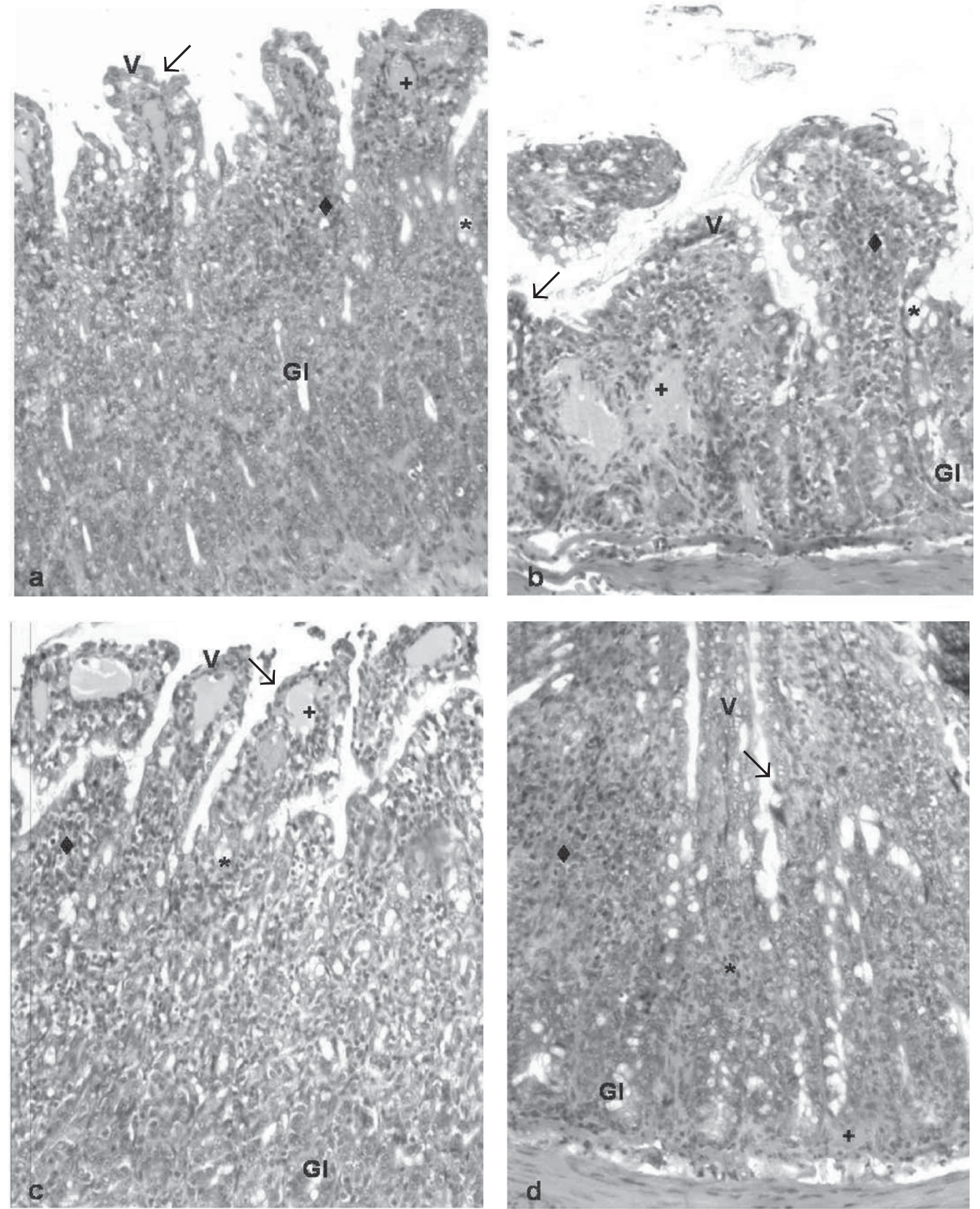

very significant and an increase in the inflammatory infiltration was observed in the lamina propria (data not shown). In group $3 \mathrm{~B}$, ileal villi were lost in some areas and the height of some villi decreased markedly. Furthermore, the number of goblet cells was approximately similar to that of the control groups (Figure 5b). 
In group 4A, the number of goblet cells decreased after melatonin treatment, the inflammation in the lamina propria was reduced and the structure was similar to that of the control groups (data not shown). In group 4B, melatonin treatment prior to TB irradiation applied in the evening did not prevent the inflammation in the lamina propria and capillary dilatation was significant in the villus apex, confirming the lack of the antiinflammatory role of melatonin in this group (Figure 5c).

The results of the present study indicate that melatonin, either in the morning or evening, showed a more prominent antiinflammatory effect after TB irradiation. The influence of melatonin administration on MPO levels also supports this finding. In groups $5 \mathrm{~A}$ (data not shown) and $5 \mathrm{~B}$, the epithelial structure was re-organized with abundant goblet cells, ileal villus loss was prevented and capillary dilatation was not detected in the lamina propria and the distribution of the lymphocytes in the lamina propria was similar to that in the control groups (Figure 5d).

These histopathological findings also confirm the protective role of melatonin in rats after AP irradiation irrespective of the time of administration, as shown by MPO levels in the present study except in rats undergoing TB irradiation in the evening.

\section{Discussion}

Influence of radiation therapy and melatonin on thiobarbituric acid reactive substance levels according to the time of administration

In the present study, higher levels of lipid peroxidation were detected in evening-excised rat hearts since TBARS levels in the control groups were higher in the evening than in the morning (27). It has been reported that TBARS levels were higher in rats under continuous darkness when compared with an alternating light-dark cycle, while continuous light did not cause any significant changes in TBARS levels (28). Therefore, it can be concluded that rats are more prone to oxidative stress in the dark.

The results of the present study demonstrate that either TB or AP irradiation in rats causes oxidative tissue damage to the ileum associated with elevated TBARS levels, an indicator of lipid peroxidation, and administration of melatonin protects against the oxidative damage after both TB and AP irradiation either in the morning or in the evening.

The rapidly proliferating epithelia of the small intestine are highly radiosensitive (29). Although the pathogenesis of radiation enteritis is not clear, it has been suggested that reactive oxygen species may act as mediators of cell injury following ionizing radiation by activated inflammatory cells, i.e., macrophages and neutrophils (3).

Ionizing radiation has been reported to increase the plasma and ovarian TBARS levels in rats and melatonin administration significantly decreased these levels (30). The radioprotective effect of melatonin has been attributed to several mechanisms such as scavenging of free radicals, distribution throughout the organism, ability to induce the antioxidant enzymes, and high bioavailability (31). It has been reported that irradiation increases the formation of MDA, an endogenous product of lactoperoxidase in the intestine $(32,33)$. Melatonin has been shown to inhibit MDA elevation, indicating that it protects against radiation-induced intestinal injury (34), in agreement with the findings of the present study.

It has been reported that at a dose of 14.4 Gy TB irradiation causes a significant decrease in the serum and pineal levels of melatonin $30 \mathrm{~min}$ to $6 \mathrm{~h}$ post-exposure in rats (16), but in the rats irradiated up to total doses of 4.8 and 9.6 Gy melatonin levels are not significantly different from control values (16). The pineal gland is not in the region directly influenced in AP-irradiated rats and endogenous melatonin level is not influ- 
enced. Thus, endogenous melatonin synthesis was not expected to be affected in the present study even after TB irradiation, since the dose was $8 \mathrm{~Gy}$. Therefore, it can be expected that neither TB nor AP irradiation caused any changes in endogenous melatonin levels in the present study. The protective effect of melatonin on oxidative damage in AP- or TB-irradiated rats cannot be attributed to the changes in endogenous melatonin levels.

Influence of radiation therapy and melatonin on myeloperoxidase levels according to time of administration

MPO activity is a marker of tissue neutrophil accumulation in inflamed tissue (12). In the present study, MPO levels were increased and melatonin treatment caused a significant decrease in rats receiving AP and TB irradiation in the morning. Histological findings confirmed the irradiation-induced inflammation in these rats.

In the present study, the presence of increased neutrophil accumulation, as assessed by elevated MPO activity in the affected tissues, indicated that radiation-induced oxidative injury in the tissue involved the contribution of neutrophil accumulation. It has been reported that macrophages produce reactive oxygen species such as superoxide $\left(\mathrm{O}_{2}{ }^{-}\right)$, hydrogen peroxide $\left(\mathrm{H}_{2} \mathrm{O}_{2}\right)$ and hydroxyl $(\cdot \mathrm{OH})$ that contribute to the pathogenesis of diseases such as inflammation (35).

It has been reported that in the ileum of ferrets MPO activity is significantly increased 2 or 7 days following exposure to only AP irradiation (5 Gy), but is reduced after TB irradiation (12). On the other hand, it has been shown that MPO activity is increased significantly in colonic tissue at both 12 and $72 \mathrm{~h}$, and in hepatic tissue at $72 \mathrm{~h}$ following whole-body irradiation, and is reduced by melatonin in rats (34).

A single dose (10 Gy) of abdominal irra- diation applied to rats did not change MPO activity after $24 \mathrm{~h}$, but increased MPO activity in the small intestine on the third day (36). Similarly, application of $10 \mathrm{~Gy}$ to the abdominal region of rats (37) increased MPO activity in the small intestine $72 \mathrm{~h}$ after irradiation. Hence, it can be concluded that the influence of radiation on MPO depends on the type, the dose and the time-course of irradiation.

Although the influence of melatonin on MPO levels after TB or AP irradiation seems inconsistent in this study, the results agree with the histological findings.

In agreement with our findings, it has been reported that TB irradiation does not cause a significant increase in MPO activity in the rat ileum, and that melatonin treatment depresses MPO activity (33).

On the other hand, it has been reported that high pharmacological doses of melatonin may induce phase changes (38). However, it has been shown that in rats melatonin has little effect on the circadian period or phase unless the time of injection coincides exactly with the onset of animal activity. The phase relationship between the time of injection and the onset of activity onset did not vary at $0 \mathrm{~h}$ at any dosage (39). During melatonin entrainment, the onset of activity continues to be phase-locked to melatonin injection until the injection regimen is stopped, at which time rats run freely according to their own circadian period and this effect is dose-dependent (40). Furthermore, phase advances of about 30-60 min in response to single injections of $50 \mu \mathrm{g}$ melatonin/kg body weight have been reported to occur at about circadian time $2 \mathrm{~h}$ before the onset of activity in constant darkness, but not at other times (38). In the present study, melatonin was administered $1 \mathrm{~h}$ after the beginning of the resting period (1 HALO) and $1 \mathrm{~h}$ after the beginning of the activity period (13 HALO). Therefore, we did not expect a prominent effect of melatonin on the circadian phase of the rats. On the other 
hand, the present study did not investigate the circadian rhythm of TBARS and MPO activity since we examined the changes in these parameters only at two different time points.

To our knowledge, this is the first study examining the time-dependent protective effects of melatonin on irradiation-induced damage in the small intestine.

Our results imply that oxidative damage is more prominent in rats receiving $\mathrm{TB}$ and AP irradiation in the morning, and with its free radical scavenging and antioxidative properties, melatonin appears to have beneficial effects on oxidative damage caused by irradiation, irrespective of the time of administration.

In the current study, the presence of increased neutrophil accumulation, assessed by elevated MPO activity in the affected tissues, indicated that AP irradiation-induced oxidative injury in the tissue and melatonin administration with RT exerted a protective effect, especially in the morning.

Thus, reducing intestinal cell injury and inhibiting neutrophil-mediated cytotoxicity will be the goals of future therapeutic strategies. Melatonin administration together with RT might be an important tool in reaching these targets.

\section{References}

1. Rubin $P$, Johnston CJ, Williams JP, McDonald S, Finkelstein JN. A perpetual cascade of cytokines postirradiation leads to pulmonary fibrosis. Int J Radiat Oncol Biol Phys 1995; 33: 99-109.

2. Zheng H, Wang J, Koteliansky VE, Gotwals PJ, Hauer-Jensen M. Recombinant soluble transforming growth factor beta type II receptor ameliorates radiation enteropathy in mice. Gastroenterology 2000; 119: 1286-1296.

3. Agrawal A, Chandra D, Kale RK. Radiation induced oxidative stress: II. Studies in liver as a distant organ of tumor bearing mice. Mol Cell Biochem 2001; 224: 9-17.

4. Yeoh E, Horowitz M, Russo A, Muecke T, Robb T, Maddox A, et al. Effect of pelvic irradiation on gastrointestinal function: a prospective longitudinal study. Am J Med 1993; 95: 397-406.

5. Gunter-Smith PJ. Gamma radiation affects active electrolyte transport by rabbit ileum: basal $\mathrm{Na}$ and Cl transport. Am J Physiol 1986; 250: G540-G545.

6. Empey LR, Papp JD, Jewell LD, Fedorak RN. Mucosal protective effects of vitamin $\mathrm{E}$ and misoprostol during acute radiation-induced enteritis in rats. Dig Dis Sci 1992; 37: 205-214.

7. Buell MG, Harding RK. Proinflammatory effects of local abdominal irradiation on rat gastrointestinal tract. Dig Dis Sci 1989; 34: 390399.

8. Sies H. Biochemistry of oxidant stress. Angewandte Chemie (International Edition in English) 1986; 25: 1058-1071.

9. Bakan E, Taysi S, Polat MF, Dalga S, Umudum Z, Bakan N, et al. Nitric oxide levels and lipid peroxidation in plasma of patients with gastric cancer. Jpn J Clin Oncol 2002; 32: 162-166.

10. Panes J, Granger DN. Leukocyte-endothelial cell interactions: molecular mechanisms and implications in gastrointestinal disease. Gastroenterology 1998; 114: 1066-1090.

11. Kettle AJ, Winterbourn CC. Myeloperoxidase: a key regulator of neutrophil oxidant production. Redox Report 1997; 3: 3-15.

12. Freeman SL, Hossain M, MacNaughton WK. Radiation-induced acute intestinal inflammation differs following total-body versus abdominopelvic irradiation in the ferret. Int J Radiat Biol 2001; 77: 389-
395.

13. Reiter RJ, Calvo JR, Karbownik M, Qi W, Tan DX. Melatonin and its relation to the immune system and inflammation. Ann NY Acad Sci 2000; 917: 376-386.

14. Reiter RJ, Tan DX, Osuna C, Gitto E. Actions of melatonin in the reduction of oxidative stress. A review. J Biomed Sci 2000; 7: 444458.

15. Reppert SM, Weaver DR. Coordination of circadian timing in mammals. Nature 2002; 418: 935-941.

16. Ahlersova E, Pastorova B, Kassayova M, Ahlers I, Smajda B. Reduced pineal melatonin biosynthesis in fractionally irradiated rats. Physiol Res 1998; 47: 133-136.

17. Lewy AJ, Wehr TA, Goodwin FK, Newsome DA, Markey SP. Light suppresses melatonin secretion in humans. Science 1980; 210 : 1267-1269.

18. Brigagao MRPL, Colepicolo P. Activation of neutrophils is daily inhibited by saliva. Biol Rhyt Res 1998; 29: 598-605.

19. Brigagao MRPL, Barroso AS, Colepicolo P. Fluctuation of reactive oxygen species released by inflammatory cells. In: Vanden Driessche T, Guisset J-L, Petiau-de Vries G (Editors), The redox state and circadian rhythms. Dordrecht Boston-London: Kluwer; 2000. p 177-191.

20. Haus E. Chronobiology of the mammalian response to ionizing radiation. Potential applications in oncology. Chronobiol Int 2002; 19: 77-100.

21. Ulusu NN, Ozbey G, Tandogan B, Gunes A, Durakoglugil DB, Karasu C, et al. Circadian variations in the activities of 6phosphogluconate dehydrogenase and glucose-6-phosphate dehydrogenase in the liver of control and streptozotocin-induced diabetic rats. Chronobiol Int 2005; 22: 667-677.

22. Hodoglugil U, Ongun O, Guney Z, Altan N, Zengil H. Temporal variation in hepatic superoxide dismutase activity in mice. Chronobiol Int 1995; 12: 152-155.

23. Cipolla-Neto J, Abdalla DS, Markus RP, Campa A. Circadian variations of superoxide dismutase activity in the rat pineal gland. $J$ 
Neural Transm Gen Sect 1993; 92: 117-123.

24. Mihara M, Uchiyama M. Determination of malonaldehyde precursor in tissues by thiobarbituric acid test. Anal Biochem 1978; 86: 271 278.

25. Grisham MB, Hernandez LA, Granger DN. Xanthine oxidase and neutrophil infiltration in intestinal ischemia. Am J Physiol 1986; 251: G567-G574.

26. Glowick SP, Kaplan SD. Methods in enzymology. New York: N.Y. Academic Press; 1955

27. Lapenna D, De Giogia S, Mezetti A, Porreca E, Ciofani G, Marzio L, et al. Circadian variations in antioxidant defences and lipid peroxidation in the rat heart. Free Rad Res Com 12992; 17: 187-194.

28. Baran D, Paduraru I, Saramet A, Petrescu E, Haulica I. Influence of light-dark cycle alteration on free radical level in rat CNS. Rom $J$ Physiol 2000; 37: 23-38.

29. Smith $\mathrm{DH}$, DeCosse JJ. Radiation damage to the small intestine. World J Surg 1986; 10: 189-194.

30. Kaya H, Delibas N, Serteser M, Ulukaya E, Ozkaya O. The effect of melatonin on lipid peroxidation during radiotherapy in female rats. Strahlenther Onkol 1999; 175: 285-288.

31. Karbownik M, Reiter RJ. Antioxidative effects of melatonin in protection against cellular damage caused by ionizing radiation. Proc Soc Exp Biol Med 2000; 225: 9-22.

32. Erbil Y, Oztezcan S, Giris M, Barbaros U, Olgac V, Bilge H, et al. The effect of glutamine on radiation-induced organ damage. Life Sci 2005; 78: 376-382.

33. Sener G, Kabasakal L, Atasoy BM, Erzik C, Velioglu-Ogunc A
Cetinel S, et al. Ginkgo biloba extract protects against ionizing radiation-induced oxidative organ damage in rats. Pharmacol Res 2006; 53: 241-252.

34. Sener G, Jahovic N, Tosun O, Atasoy BM, Yegen BC. Melatonin ameliorates ionizing radiation-induced oxidative organ damage in rats. Life Sci 2003; 74: 563-572.

35. Klebanoff SJ. Myeloperoxidase: friend and foe. J Leukoc Biol 2005; 77: 598-625.

36. Picard C, Wysocki J, Linard C, Garcia-Villar R, Bueno L, Griffiths $\mathrm{NM}$, et al. Absence of protective role of afferent nerves in early intestinal mucosal alterations induced by abdominal irradiation in rats. Int J Radiat Biol 2001; 77: 349-356.

37. Olgac V, Erbil Y, Barbaros U, Oztezcan S, Giris M, Kaya H, et al The efficacy of octreotide in pancreatic and intestinal changes: radiation-induced enteritis in animals. Dig Dis Sci 2006; 51: 227232

38. Illnerova $\mathrm{H}$. The suprachiasmatic nucleus and rhythmic pineal melatonin production. In: Klein DC, Moore RY, Reppert SM (Editors), Suprachiasmatic nucleus: The mind's clock. New York: Oxford University Press; 1991. p 197-216.

39. Cassone VM, Chesworth MJ, Armstrong SM. Dose-dependent entrainment of rat circadian rhythms by daily injection of melatonin. $J$ Biol Rhythms 1986; 1: 219-229.

40. Armstrong SM, Chesworth JR. Melatonin phase-shifts a mammalian circadian clock. In: Trentini GP, De Gaetani C, Pevet P (Editors), Fundamentals and clinics in pineal research. New York: Raven Press; 1987. p 195-198. 